\title{
22. INSOLUBLE ORGANIC MATTER BITUMENS IN LEG 47 SAMPLES
}

\author{
D. L. Johnson, R. D. McIver, ${ }^{1}$ and M. A. Rogers, Exxon Production Research Company Houston, Texas
}

\section{SUMMARY}

Analyses of 86 core samples from Holes 397, 397A, 398A, and 398D of Leg 47 of the Deep Sea Drilling Project point up the erratic contents of organic matter, particularly in the sediments at Site 398 . The majority of the samples from this site near Spain contain predominantly woody and coaly organic matter, in contrast to the generally more algal, amorphous, and herbaceous organic matter of Site 397 off northwestern Africa. Unexpectedly, thermal alteration index of this material, determined visually by standard microscopic techniques, do not show a trend of longer apparent exposure to increased temperatures at depth, perhaps suggesting mixing of sediment from different provenances.

The bitumens in some of the Site 398 samples might be interpreted as immature oil sources. Their immaturity further is confirmed by their low hydrocarbon contents: all below $50 \mathrm{ppm}$, and most below $5 \mathrm{ppm}$. While the Site 398 bitumens appear similar to Site 397 extracts in degree of maturity, samples from the latter are consistently richer in bitumens. Together with the greater algal and amorphous nature of samples from Site 397, this bitumen-richness further points up that with prolonged exposure to higher temperatures, sediments such as those surrounding Site 398 would be primarily sources of gas, perhaps with liquids, whereas those sediments found contiguous to Site 397 would be probable oil sources.

\section{INTRODUCTION}

As a continuing program, occasional samples are collected for organic geochemical studies. Some are taken by the shipboard geochemist for his own studies; and others are frozen, shipped to the sample repository, and later distributed to a panel of organic geochemists. The samples herein reported are about equally split between the above two types. In this case, the second group was selected to fill in sample coverage between those taken aboard ship by one of the authors (D. L. Johnson).

We submitted the cores for standard analyses (McIver, 1974). First the samples were cleaned by removing a thin film from the outside surfaces and then were dried in a dust-free hood. The dried sample was pulverized and homogenized. A small portion was removed from each for determination of organic carbon after carbonate removal with $1 N$ hydrochloric acid.

'GeoChem Research Inc., Houston, Texas.
Another aliquot was submitted for visual description of the kerogen by microscopy; it was treated first with $\mathrm{HCl}$ and $\mathrm{HF}$ to remove carbonates and silicates, respectively. The remaining portions were extracted with organic solvents to remove the soluble bitumens, from which the hydrocarbons were separated by liquid-solid chromatography.

Results of these analyses are presented in Table 1 for samples from Sites 397 and 398. Figure 1 graphically provides the organic geochemical profile of Site 398.

\section{RESULTS AND INTERPRETATION}

Hole 397: The five samples, ranging in age from Quaternary to early Miocene, all have only modest amounts of organic matter. However, the bitumen contents are high with respect to the organic contents. This is consistent with the fact that these samples contain small amounts of amorphous and algal kerogen and significant fractions of the herbaceous type. The erratic alteration indexes suggest that much of the kerogen in the uppermost sample may be reworked organic detritus. These sediments are interpreted as being immature, and oil-prone, but low in ultimate hydrocarbongenerating potential.

Hole 397A: The five samples from this hole range in age from early Miocene (the upper four) to Early Cretaceous (the lowermost). Four of the five are moderately rich in organic carbon, and the four Tertiary ones, like those in the younger sediments sampled in Hole 397, contain oil-favoring algal organic matter. The Cretaceous sample is mostly woody and (to a lesser extend) herbaceous, which suggests a gas-prone nature. However, none of this potential has been realized by these sediments because they have experienced such gentle time-temperature histories.

Holes 398A and 398D: As the table and figure show, the organic matter contents and types in the Miocene and Cretaceous sediments at this locale are erratic. Yet, there is a predominance of woody and coaly organic matter in them, suggesting a gassy (i.e., gasprone) facies. There are a few exceptions (e.g., Sections $56-2,75-2,97-2$, and 130-5) in which algal and amorphous kerogen types make up from 40 to 90 per cent of the kerogen. These apparently oil-prone laminations are the product of temporary changes in environment of deposition and perhaps differences in provenance.

The samples are generally lean in organic matter down to the early Cenomanian (Section 56-2) where the first occurrence of very rich dark gray to black algal zones is noted. Below this depth, the organic contents generally increase, and much of the earlier Creta- 
TABLE 1

Organic Geochemistry of Leg 47 Cores

\begin{tabular}{|c|c|c|c|c|c|c|c|c|c|c|}
\hline \multirow{2}{*}{$\begin{array}{c}\text { Sample } \\
\text { (Interval in } \mathrm{cm} \text { ) }\end{array}$} & \multirow{2}{*}{$\begin{array}{l}\text { Sub-Bottom } \\
\text { Depth } \\
\text { (m) }\end{array}$} & \multirow{2}{*}{$\begin{array}{l}\text { Organic } \\
\text { Carbon } \\
\text { (wt. \%) }\end{array}$} & \multirow{2}{*}{$\begin{array}{l}\text { Bitumen } \\
\text { (ppm) }\end{array}$} & \multirow{2}{*}{$\begin{array}{c}\mathrm{C}_{15+} \\
\text { Hydro- } \\
\text { Carbons } \\
\text { (ppm) }\end{array}$} & \multicolumn{5}{|c|}{ Kerogen Types (in tenths) } & \multirow{2}{*}{$\begin{array}{c}\text { Thermal } \\
\text { Alteration } \\
\text { Index }\end{array}$} \\
\hline & & & & & Algal & Amorphous & Herbaceous & Woody & Coaly & \\
\hline \multicolumn{11}{|l|}{ Hole 397} \\
\hline $10-6,35-50$ & 92.9 & 0.32 & 256 & $\mathrm{TR}^{\mathrm{a}}$ & $\operatorname{Tr}^{\mathrm{b}}$ & - & 1 & 5 & 3 & $3-$ \\
\hline $18-5,110-130$ & 168.2 & 0.48 & 480 & TR & $\operatorname{Tr}$ & - & 2 & 5 & 3 & 1 \\
\hline $32-2,125-137$ & 296.8 & 0.23 & 382 & TR & $\operatorname{Tr}$ & - & - & 6 & 3 & 1 \\
\hline $52-3,100-130$ & 497.7 & 0.12 & 583 & TR & - & $\mathrm{Tr}$ & 1 & 5 & 4 & 1 \\
\hline $95-4,100-130$ & 907.7 & 0.36 & 239 & TR & 1 & - & 3 & 6 & - & $2-$ \\
\hline \multicolumn{11}{|l|}{ Hole 397A } \\
\hline $12-2,0-20$ & 1031.6 & 1.14 & 417 & 23 & 4 & $\operatorname{Tr}$ & 1 & 3 & - & $1+$ \\
\hline $16-4,0-20$ & 1072.6 & 1.36 & 540 & 66 & 3 & - & 2 & 5 & - & $1+$ \\
\hline $21-2,120-150$ & 1118.4 & 0.25 & 286 & TR & 2 & - & 3 & 5 & - & $2-$ \\
\hline $23-4,25-36$ & 1158.4 & 1.24 & 431 & 63 & 6 & - & - & 2 & - & $1+$ \\
\hline $51-4,130-140$ & 1444.0 & 0.83 & 834 & 17 & - & - & 2 & 8 & - & $1+$ \\
\hline \multicolumn{11}{|l|}{ Hole 398A } \\
\hline \multicolumn{11}{|l|}{ Hole 398D } \\
\hline $2-2,50-52$ & 272.8 & 0.34 & - & - & - & - & 3 & 4 & 3 & 1 \\
\hline $2-2,70-72$ & 273.0 & 0.09 & - & - & - & - & - & 6 & 4 & 2 \\
\hline $3-7,40-42$ & 327.6 & 0.11 & - & - & - & - & - & 8 & 2 & $2+$ \\
\hline $4-3,70-72$ & - & 0.05 & - & - & - & - & - & 8 & 2 & $2+$ \\
\hline $6-4,130-140$ & 419.2 & 0.13 & 505 & TR & - & 1 & - & 6 & 3 & $1+$ \\
\hline $6-6,106-108$ & 421.8 & 0.12 & - & - & - & - & - & 9 & 1 & $2+$ \\
\hline $9-3,77-79$ & 474.2 & 0.05 & - & - & - & - & - & 8 & 2 & $2-$ \\
\hline $12-2,16-17$ & 509.0 & 0.07 & - & - & - & - & - & 9 & 1 & $2+$ \\
\hline $19-3,130-150$ & 588.9 & 0.03 & 138 & TR & - & - & - & - & - & - \\
\hline $19-4,38-40$ & 589.1 & 0.05 & - & - & - & - & - & - & - & - \\
\hline $23-4,34-36$ & 637.3 & 0.06 & - & - & - & - & - & - & - & - \\
\hline $27-1,111-118$ & 661.3 & 0.06 & - & - & - & - & - & - & - & - \\
\hline $28-3,23-25$ & 673.0 & 0.04 & - & - & - & - & - & - & - & - \\
\hline $31-3,130-150$ & 702.4 & 0.05 & 119 & TR & - & - & - & - & - & - \\
\hline $33-2,125-127$ & 720.2 & 0.07 & - & - & - & - & - & - & - & - \\
\hline $35-2,109-111$ & 739.1 & 0.07 & - & - & - & - & - & - & - & - \\
\hline $39-1,39-41$ & 774.7 & 0.09 & - & - & - & - & - & - & - & - \\
\hline $40-4,120-140$ & 789.9 & 0.06 & 238 & TR & - & - & - & - & - & - \\
\hline $47-4,29-31$ & 855.2 & 0.05 & - & - & - & - & - & - & - & - \\
\hline $48-4,40-42$ & 864.7 & 0.05 & - & - & - & - & - & - & - & - \\
\hline $53-4,120-150$ & 913.3 & 0.09 & 95 & TR & - & - & - & - & - & - \\
\hline $55-1,33-35$ & 926.5 & 0.04 & - & - & - & - & - & - & - & - \\
\hline $56-2,25$ & 947.2 & $4: 56$ & - & - & 9 & - & - & $\operatorname{Tr}$ & $\operatorname{Tr}$ & 1 \\
\hline Comp. $^{\mathrm{c}}$ & $945-974$ & 0.43 & 92 & TR & 1 & - & 1 & 5 & 3 & 1 \\
\hline $\begin{array}{c}57-4, \begin{array}{c}145-150 \& \& \\
130-135\end{array}\end{array}$ & 960.9 & 0.39 & 171 & TR & - & - & 4 & 3 & 3 & $2-$ \\
\hline $59-6,149-150$ & 982.9 & 0.60 & - & - & $\operatorname{Tr}$ & - & 3 & 3 & 3 & $2-$ \\
\hline $62-4,120-135$ & 1008.3 & 0.41 & 136 & TR & - & - & 2 & 4 & 4 & 2 \\
\hline $62-7,25-27$ & 1011.6 & 0.32 & - & - & - & - & 1 & 7 & 2 & $2-$ \\
\hline $65-2,61-63$ & 1032.9 & 0.36 & - & - & - & - & 1 & 7 & 2 & 1 \\
\hline $65-4,110-130$ & 1036.7 & 0.24 & 223 & TR & - & - & 1 & 6 & 3 & $2+$ \\
\hline Comp. & $1059-1069$ & 0.90 & 117 & 42 & - & - & 2 & 5 & 3 & 1 \\
\hline Comp. & $1064-1082$ & 0.70 & 137 & TR & - & - & 3 & 5 & 2 & $1+$ \\
\hline $69-2,112-120$ & 1071.8 & 0.57 & 146 & TR & 2 & - & 1 & 4 & 3 & 2 \\
\hline $71-3,103-105$ & 1092.0 & 0.46 & - & - & - & - & 1 & 7 & 2 & $1+$ \\
\hline Comp. & $1098-1173$ & 0.70 & 108 & TR & - & - & 2 & 6 & 2 & $1+$ \\
\hline $73-4,120-150$ & 1122.4 & 0.62 & 167 & TR & - & - & 2 & 5 & 3 & $2-$ \\
\hline $75-2,110-130$ & 1138.2 & 2.69 & 926 & 43 & 4 & - & - & 3 & 3 & $2+$ \\
\hline Comp. & $1175-1177$ & 0.78 & 118 & 29 & - & - & - & - & - & - \\
\hline Comp. & $1183-1189$ & 0.77 & 81 & TR & - & - & 1 & 6 & 3 & $1+$ \\
\hline Comp. & $1192-1194$ & 0.93 & 131 & 20 & $\mathrm{Tr}$ & - & 2 & 5 & 3 & $1+$ \\
\hline $81-2,50-80$ & 1204.2 & 1.06 & 266 & TR & $\operatorname{Tr}$ & - & 3 & 3 & 3 & 2 \\
\hline $82-3,75-77$ & 1215.2 & 0.42 & - & - & - & - & 1 & 7 & 2 & $2-$ \\
\hline $84-4,15-30$ & 1235.2 & 0.83 & 148 & TR & - & - & 3 & 4 & 3 & 2 \\
\hline $85-1,53-55$ & 1240.5 & 0.43 & - & - & - & - & $\operatorname{Tr}$ & 7 & 2 & $2-$ \\
\hline $86-4,50-100$ & 1254.7 & 1.00 & 267 & TR & - & - & 3 & 4 & 3 & 2 \\
\hline Comp. & $1251-1261$ & 1.23 & 108 & 15 & 1 & - & 3 & 4 & 2 & $1+$ \\
\hline
\end{tabular}


TABLE 1 - Continued

\begin{tabular}{|c|c|c|c|c|c|c|c|c|c|c|}
\hline \multirow{2}{*}{$\begin{array}{c}\text { Sample } \\
\text { (Interval in } \mathrm{cm} \text { ) }\end{array}$} & \multirow{2}{*}{$\begin{array}{l}\text { Sub-Bottom } \\
\text { Depth } \\
\text { (m) }\end{array}$} & \multirow{2}{*}{$\begin{array}{l}\text { Organic } \\
\text { Carbon } \\
\text { (wt. \%) }\end{array}$} & \multirow{2}{*}{$\begin{array}{l}\text { Bitumen } \\
\text { (ppm) }\end{array}$} & \multirow{2}{*}{$\begin{array}{c}\mathrm{C}_{15+} \\
\text { Hydro- } \\
\text { Carbons } \\
\text { (ppm) }\end{array}$} & \multicolumn{5}{|c|}{ Kerogen Types (in tenths) } & \multirow{2}{*}{$\begin{array}{l}\text { Thermal } \\
\text { Alteration } \\
\text { Index }\end{array}$} \\
\hline & & & & & Algal & Amorphous & Herbaceous & Woody & Coaly & \\
\hline $87-2,150-155$ & 1261.8 & 1.13 & - & - & - & $\mathrm{Tr}$ & 5 & 3 & 2 & $1+$ \\
\hline $88-4,62-95$ & 1273.8 & 0.78 & 71 & TR & - & - & $\operatorname{Tr}$ & 7 & 2 & $2+$ \\
\hline $90-5,0-15$ & 1293.6 & 0.63 & 98 & TR & - & - & 2 & 5 & 3 & $2-$ \\
\hline Comp. & $1327-1331$ & 1.87 & 152 & 16 & - & - & 3 & 4 & 3 & $1+$ \\
\hline $95-3,18-35$ & 1330.8 & 1.32 & 193 & TR & - & - & 3 & 4 & 3 & $2-$ \\
\hline $96-2,40-42$ & 1326.8 & 2.32 & - & - & 2 & 2 & $\operatorname{Tr}$ & 3 & 2 & $1+$ \\
\hline $97-3,0-25$ & 1347.6 & 1.25 & 112 & TR & - & - & 1 & 5 & 4 & $2+$ \\
\hline $99-4,25-55$ & 1368.4 & 0.95 & 67 & TR & - & - & 2 & 5 & 2 & 2 \\
\hline Comp. & $1345-1377$ & 1.31 & 77 & TR & - & - & 2 & 5 & 3 & $1+$ \\
\hline $101-1,145-147$ & 1383.7 & 0.76 & - & - & - & - & $\operatorname{Tr}$ & 9 & 1 & $1+$ \\
\hline $104-2,88-91$ & 1413.3 & 0.53 & - & - & $\operatorname{Tr}$ & - & 2 & 5 & 2 & $1+$ \\
\hline $105-3,145-147$ & 1424.9 & 0.45 & - & - & - & - & 1 & 8 & 1 & $1+$ \\
\hline $106-3,47-49$ & 1433.4 & 0.51 & - & - & - & - & 2 & 6 & 2 & $1+$ \\
\hline $106-5,100-150$ & 1437.3 & 0.51 & 72 & TR & $\mathrm{Tr}$ & - & 1 & 6 & 2 & 2 \\
\hline $107-2,40-42$ & 1441.0 & 0.47 & - & - & - & - & 1 & 8 & 1 & $1+$ \\
\hline $108-3,106-113$ & 1453.1 & 0.43 & 111 & TR & - & - & 3 & 4 & 3 & $2-$ \\
\hline $111-5,96-98$ & 1484.3 & 0.54 & - & - & - & - & 2 & 6 & 2 & $1+$ \\
\hline $112-2,0-30$ & 1488.6 & 0.34 & 81 & TR & - & - & 1 & 5 & 4 & $1+$ \\
\hline Comp. & $1496-1555$ & 0.81 & 107 & TR & $\operatorname{Tr}$ & - & 3 & 3 & 3 & $1+$ \\
\hline $114-3,40-47$ & 1509.4 & 0.38 & 76 & TR & - & - & 3 & 6 & 1 & $1+$ \\
\hline $118-5,0-15$ & 1550.1 & 0.73 & 135 & TR & - & - & $\operatorname{Tr}$ & 7 & 2 & $2-$ \\
\hline $120-3,89$ & 1566.6 & 0.87 & - & - & 1 & - & 2 & 5 & 2 & $1+$ \\
\hline Comp. & $1579-1625$ & 2.71 & 293 & 11 & $\operatorname{Tr}$ & - & 1 & 6 & 2 & $1+$ \\
\hline $122-5,142-150$ & 1589.5 & 2.40 & 655 & 33 & $\mathrm{Tr}$ & - & 1 & 6 & 3 & $2-$ \\
\hline $127-4,61-63$ & 1634.3 & 2.51 & - & - & - & - & 1 & 8 & 1 & $2-$ \\
\hline $128-1,17-19$ & 1638.8 & 2.31 & - & - & - & - & $\operatorname{Tr}$ & 8 & 2 & $2-$ \\
\hline Comp. & $1651-1736$ & 1.48 & 148 & TR & $\mathrm{Tr}$ & - & 1 & 7 & 2 & $1+$ \\
\hline $130-5,126-134$ & 1665.3 & 2.07 & 114 & TR & 6 & - & $\mathrm{Tr}$ & 1 & - & $2-$ \\
\hline $138-2,53-55$ & 1735.8 & 0.66 & - & - & $\mathrm{Tr}$ & $\operatorname{Tr}$ & 3 & 3 & 3 & 1 \\
\hline
\end{tabular}

ceous sediments have enough organic matter to be classed a potential petroleum (mostly gas) source rock. One of the very dark clay zones yielded samples with organic and algal kerogen contents, that are higher, as the oiliness of the sediments. In the more abundant lighter zones, the organic content is generally lower and the woody-coaly kerogen types predominate.

The Barremian samples are uniformly organic-rich, but are very coaly, and thus gassy.

The samples from Site 398 are almost uniformly immature. The thermal alteration indexes are erratic, but most are below the threshold for significant hydrocarbon generation.

The erratic nature of the measured parameters is a reflection of the rapidly changing and cyclic environ- ment of deposition and organic source. The abundance of woody-coaly kerogen at least suggests a long-lived source of continental organic debris supplying coaly and woody material to this depositional site. Such an environment encountered in more deeply buried (i.e., more thermally mature) sediments would be expected to generate large quantities of gaseous and only modest quantities of liquid hydrocarbons.

\section{REFERENCE}

McIver, R. D., 1974. Evidence of migrating hydrocarbons in Deep Sea Drilling Project cores, Am. Assoc. Petrol. Geol. Bull., v. 58 , No. 7. p. 1263-1271. 
D. L. JOHNSON, R. D. McIVER, M. A. ROGERS

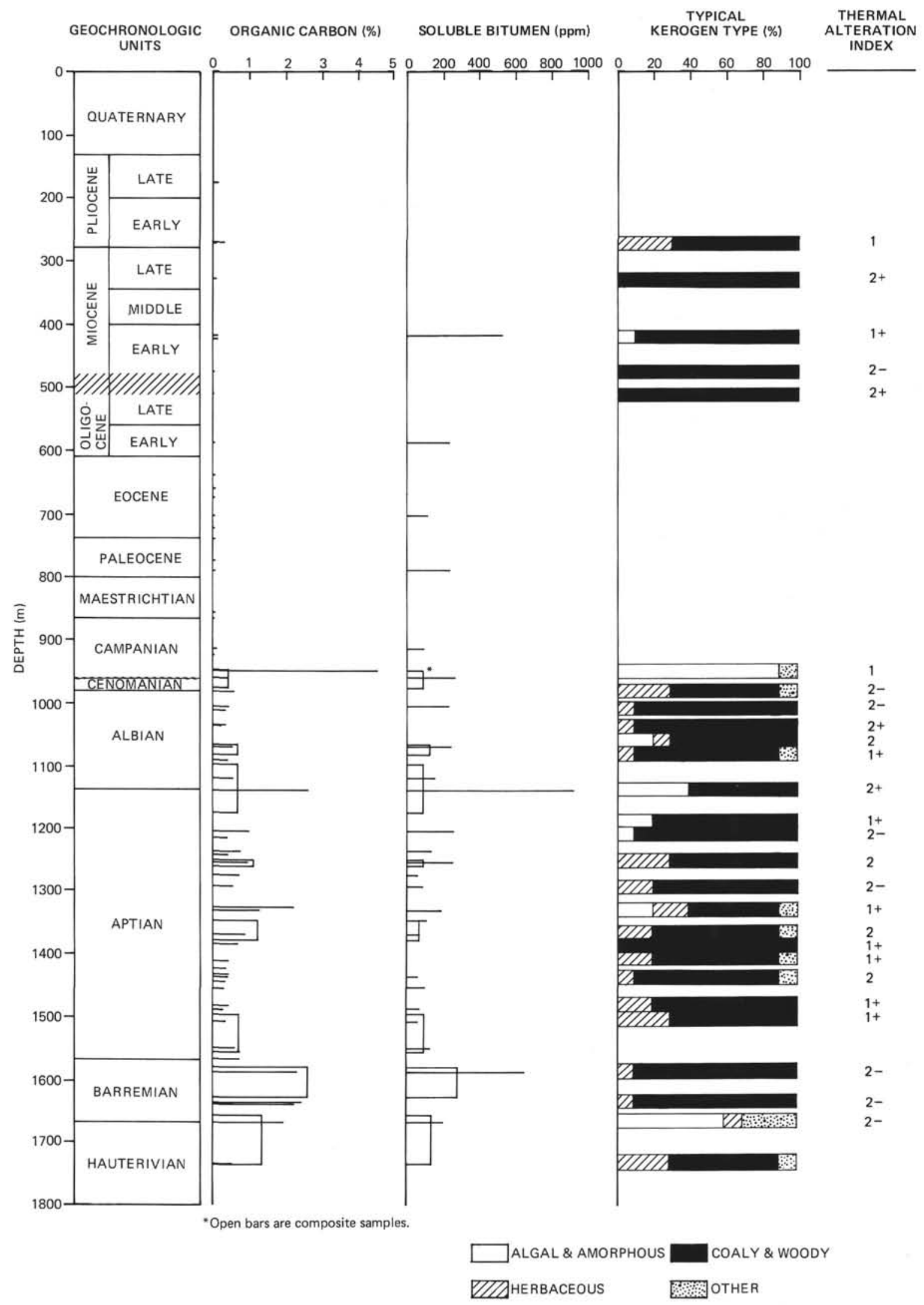

Figure 1. Organic geochemistry of Site 398. 